\title{
Dynamic asymmetries in house price cycles: A generalized smooth transition model
}

\author{
Alessandra Canepa ${ }^{\mathrm{a}, *}$, Emilio Zanetti Chini ${ }^{\mathrm{b}}$ \\ a Brunel University London, UK \\ ${ }^{\mathrm{b}}$ University of Pavia, Italy
}

\section{A R T I C L E I N F O}

\begin{tabular}{l}
\hline Article history: \\
Received 11 September 2015 \\
Received in revised form 22 February 2016 \\
Accepted 25 February 2016 \\
Available online 8 March 2016 \\
\hline JEL Classification: \\
C10 \\
C31 \\
C33 \\
Keywords: \\
House price cycles \\
Dynamic asymmetries \\
Non-linear models \\
Forecasting
\end{tabular}

\begin{abstract}
A B S T R A C T
In this paper we propose a novel nonlinear model to capture asymmetries in real estate cycles. The approach involves a particular parametrization of the transition function used in the transition equation of a smooth transition autoregressive model which improves the fit in the non-central probability region. The dynamic symmetry in house price cycles is strongly rejected for the housing markets taken into consideration. Further, our results show that the proposed model performs well in a out of sample forecasting exercise.
\end{abstract}

(C) 2016 The Authors. Published by Elsevier B.V. This is an open access article under the CC BY-NC-ND license (http://creativecommons.org/licenses/by-nc-nd/4.0/)

\section{Introduction}

Modelling the behavior of housing markets has always been an important issue for academics due to spillover effects on the whole economy. Accurate modelling of house prices is also crucial for policy makers interested in preventing unsustainable swings in the housing markets. However, modeling house price appreciation proved to be a challenging task due to the strong vulnerability of the housing markets to finance systems and market fundamentals.

A large body of literature shows that real estate cycles are closely related to business cycles. From the theoretical point of view, in the literature models used to describe housing market cycles fall within the demand-supply framework, where supply is assumed to be rigid. For example, Abraham and Hendershott (1996) describe an equilibrium price level to which the housing market tends to adjust. The authors divide the determinants of house price appreciation in two groups: one that

\footnotetext{
* Corresponding author at: Department of Economics and Finance, Brunel University London, Uxbridge, Middlesex UB8 3PH, United Kingdom UK. Tel.: $+44(0) 1895266207$

E-mail address: Alessandra.Canepa@brunel.ac.uk (A. Canepa).
} 
explains changes in the equilibrium price and another that accounts for the adjustment mechanism in the equilibrium process. Slow adjustment toward the equilibrium can be regarded as an indication of asymmetries in real estate cycles. Muellbauer and Murphy (1997) explore the behaviour of house prices in the UK. The authors suggest that the presence of transaction costs associated with the housing market cause important nonlinearity in house price dynamics. Further, Holly et al. (2010) (see also Holly et al., 2011) extend the analysis to the spatiotemporal diffusion of shocks in the housing market. Using a model that takes into account spatial and temporal interactions the authors are able to investigate to which extent real house prices are driven by common shocks.

Another strand of the literature has related asymmetric cyclical movements of housing markets to house price bubbles. In their seminal paper Case and Shiller (1989) find that house prices are correlated, which suggests that residential property markets are inefficient. In a more recent work Case and Shiller (2003) consider house prices in relation to the fundamentals. The authors make a compelling case that house prices exhibit statistically significant short-term momentum and do not incorporate information about changes in the fundamentals.

The purpose of this paper is to seek empirical evidence of asymmetric behaviour in house prices cycles. To this end a novel regime-switching model is proposed in order to capture dynamic asymmetries in the housing market. Although almost all previous empirical work relating to house price modelling is based on linear specifications (see for example Abraham and Hendershott, 1996) the well established literature on cyclical behavior of macroeconomic variables suggests that the nonlinearity of house prices should stem from the asymmetric properties of house price determinants like GDP, interest rate or bank lending. There are a few available empirical works which corroborate this hypothesis; for example, Kim and Bhattacharya (2009) use smooth a transition autoregressive model (STAR) to test for nonlinearity in the regional hosing market in the United States. Balcilar et al. (2015) use a STAR-type model to forecast house price distributions. Nonlinear models are also used in Crawford and Fratantoni (2003) to forecast house price changes.

Regime-swiching models such as STAR allow the dynamic of house price growth rates to evolve according to a smooth transition between regimes that depends on the sign and magnitude of past realization of house price growth rates (see Chan and Tong (1986)). The low speed of transition between different regimes in house price growth found in empirical studies validate the choice of smooth transition models. A possible shortcoming of this type of nonlinear models is that a symmetric transition function is used to capture oscillations from the conditional mean of the changes in the house price series. Although STAR-type models efficiently describe nonlinearity in house price growth rates, the most commonly used transition functions may not be suitable to capture dynamic asymmetries in real estate cycles.

In this paper we address the following questions: Are contractions in house prices steeper than expansions, or does the amplitude of troughs in house prices exceeds that of peaks? From the methodological point of view the question is as follows: Is the rate of change in the left tail of the transition function of the STAR-type model different with respect to that of the right tail? And if so, how much? We argue that being the transition function adopted in STAR-type models generally symmetric, by construction, the resulting model may not be suitable to address the issues above. We believe that what economic literature has called "asymmetry" may be better reflected by a statistical model that uses a more general parametrization of the transition function. Accordingly, in this work it is suggested that a class of models indexed by two shape parameters be used to model dynamic asymmetry in house price cycles. By using shape parameters that influence the symmetry and heaviness of the tails of the fitted transition equation the proposed model may be more suitable to fit the non-central probability regions and therefore be better able to capture the asymmetries that are often found in empirical research. The suggested transition function, which is in the spirit of Stukel (1988), has two parameters governing the two tails of the sigmoid function in the nonlinear component of the model. The advantage of the proposed parameterization is that the resulting model is able to preserve smoothness of the transition function without requiring any restriction in the parameters. This feature may be appealing in empirical applications. Below we refer to the proposed model as generalized smooth transition autoregressive model (GSTAR).

Asymmetric behaviour over the business cycle has long been the object of interest in applied and theoretical research. It is therefore not surprising that several variations of the STAR model have been suggested in the literature. For example, Sollis et al. (1999) suggest to raise the transition function of the STAR to an exponential. Alternatively, Sollis et al. (1999) propose to add a parameter inside the transition function in order to control the asymmetry of both tails of the transition function. The suggested procedures successfully address the issue of dynamic asymmetry in several classical macroeconomic series. A possible shortcoming, however, is that the transition function suggested by Sollis et al. (2002) may be non-smooth. Also, the effect of increasing the asymmetry of the parameter may resolve to a shift of the transition function (see Zanetti Chini (2014) and Lundbergh and Teräsvirta (2006)).

Our results reveal several insights into the patterns of the housing markets under consideration. In particular, it is found that during boom periods house prices deviate from their mean at an exponential rate, whereas they return to the equilibrium level at a logarithmic rate. This implies that when improving economic conditions boost housing demand above the potential stock, prices rise rapidly above their expected level. On the other hand, house prices fall slowly when economic conditions worsen and changes in house prices are below the expected values. We then consider the forecasting properties of the GSTAR model by comparing the out of sample performance of the GSTAR with an autoregressive linear model. Using several performance criteria it is found that, overall, the proposed nonlinear model performs better than its symmetric counterpart.

The reminder of this paper proceeds as follows. In Section 2 the specification, estimation and testing of the proposed nonlinear model is presented. In Section 3 the empirical results are described. Section 4 offers some concluding remarks. In the Appendix the derivations for the misspecification tests used in Section 3 are reported. 


\section{The generalized smooth transition model}

Let $y_{t}$ be a realization of a the house price series observed at $t=1-p, 1-(p-1), \ldots,-1,0,1, T-1, T$. Then, the univariate process $\left\{y_{t}\right\}_{t}^{T}$ can be specified using the following model

$$
\begin{aligned}
& y_{t}=\phi^{\prime} z_{t}+\theta^{\prime} z_{t} G\left(\gamma, h\left(c_{k}, s_{t}\right)\right)+\epsilon_{t}, \quad \epsilon_{t} \sim \text { I.I.D. }\left(0, \sigma^{2}\right) \\
& G\left(\gamma, h\left(c_{k}, s_{t}\right)\right)=\left(1+\exp -\left\{\prod_{k=1}^{K} h\left(c_{k}, s_{t}\right)\right\}\right)^{-1}, \\
& h\left(c_{k}, s_{t}\right)=\left\{\begin{array}{ll}
\gamma_{1}^{-1} \exp \left(\gamma^{-1}\left|s_{t}-c_{k}\right|-1,\right. & \text { if } \gamma_{1}>0 \\
s_{t}-c_{k}, & \text { if } \gamma_{1}=0 \\
-\gamma_{1}^{-1} \log \left(1-\gamma_{1}\left|s_{t}-c_{k}\right|\right), & \text { if } \gamma_{1}<0
\end{array}\right\}
\end{aligned}
$$

for $\left(s_{t}-c_{k}\right)>0$ (or, equivalently, $\left.h\left(c, s_{t}\right)>1 / 2\right)$ and

$$
h\left(c_{k}, s_{t}\right)=\left\{\begin{array}{ll}
\gamma_{2}^{-1} \exp \left(\gamma^{-1}\left|s_{t}-c_{k}\right|-1,\right. & \text { if } \gamma_{2}>0 \\
s_{t}-c_{k}, & \text { if } \gamma_{2}=0 \\
-\gamma_{2}^{-1} \log \left(1-\gamma_{2}\left|s_{t}-c_{k}\right|\right), & \text { if } \gamma_{2}<0
\end{array}\right\}
$$

for $\left(s_{t}-c_{k}\right) \leq 0$ (or, equivalently, $h\left(c_{k}, s_{t}\right)<1 / 2$ ).

In Eqs. (1)-(4) the vectors $z_{t}=\left(1, y_{t-1}, \ldots, y_{t-p}\right)^{\prime}, \phi=\left(\phi_{0}, \phi_{1}, \ldots, \phi_{p}\right)^{\prime}, \theta=\left(\theta_{0}, \theta_{1}, \ldots, \theta_{p}\right)^{\prime}$ are parameter vectors. The process $\left\{\epsilon_{t}\right\}_{t}^{T}$ in Eq. (1) is assumed to be a martingale difference sequence with respect to the history of the time series up to time $t-1$, denoted as $\Omega_{t-1}=\left[y_{1-(p-a)}, y_{t-p}\right]$, with $E\left[\epsilon_{t} \mid \Omega_{t-1}\right]=0$ and $E\left[\epsilon_{t}^{2} \mid \Omega_{t-1}\right]=\sigma^{2}$. The expression $G\left(\tilde{\gamma}, h\left(c_{k}, s_{t}\right)\right)$ defines the transition function, which is assumed to be continuously differentiable with respect to the scale parameters $\tilde{\gamma} \in\left(\gamma_{1}, \gamma_{2}\right)$ and bounded between 0 and 1 . Also, $G\left(\tilde{\gamma}, h\left(c_{k}, s_{t}\right)\right)$ is continuous in the function $h\left(c_{k}, s_{t}\right)$ and $h\left(c_{k}, s_{t}\right)$ is strictly increasing in the transition variable $s_{t}$. The transition variable $s_{t}$ is assumed to be a lagged endogenous variable, that is, $s_{t}=y_{t-d}$ for a certain integer $d>0$. Note that $s_{t}$ can also be an exogenous variable or possibly a function of lagged endogenous variables. The parameters $c_{k} \in\{1,2\}$ are the location parameters.

To simplify the notation in Eqs. (2)-(4) it is convenient to denote the kernel of the model corresponding to the $k$-th location as $\eta_{k, t} \equiv s_{t}-c_{k}$. Therefore, the transition function in Eqs. (2)-(4) can be written as

$$
G\left(\tilde{\gamma}, h\left(\eta_{k, t}\right)\right)=\left(1+\exp \left\{\prod_{k=1}^{K}\left[\begin{array}{l}
h\left(\eta_{k, t}\right) I_{\left(\gamma_{1} \leq 0, \gamma_{2} \leq 0\right)}+h\left(\eta_{k, t}\right) I_{\left(\gamma_{1} \leq 0, \gamma_{2}>0\right)}+ \\
+h\left(\eta_{k, t}\right) I_{\left(\gamma_{1}>0, \gamma_{2} \leq 0\right)}+h\left(\eta_{k, t}\right) I_{\left(\gamma_{1}>0, \gamma_{2}>0\right)}
\end{array}\right]\right\}\right)^{-1} .
$$

Asymmetric behaviour in house price dynamics is introduced in the model by Eqs. (3)-(4). In particular, Eq. (3) models the higher tail of the probability function, whereas Eq. (4) models the lower tail of the probability function. The velocity of the transition function is controlled by the slope parameters $\tilde{\gamma}$. In Eq. (5) if $\tilde{\gamma}>0$, the function $h\left(\eta_{k, t}\right)$ is an exponential rescaling which increases more quickly than a standard logistic function. On the other hand, if $\tilde{\gamma}<0$, the function $h\left(\eta_{k, t}\right)$ is a logarithmic rescaling which increases more slowly than a standard logistic function. The case with $h\left(\eta_{k, t}\right)=\eta_{k, t}$ implies that the function nests a one-parameter symmetric logistic STAR model (see Teräsvirta (1994)) with slope $\gamma_{1}=\gamma_{2}=\gamma$.

Different choices of the transition function $G\left(\tilde{\gamma}, h\left(c_{k}, s_{t}\right)\right)$ give rise to different types of regime-switching behaviour. If $k=1$ in Eq. (2) the parameters on the right hand side of Eq. (1) change monotonically as a function of $s_{t}$ from $\phi$ to $\phi+\theta$ and the corresponding transition function is given by

$$
G\left(\tilde{\gamma}, h\left(\eta_{1, t}\right)\right)=\left(1+\exp \left\{-h\left(\eta_{1, t}\right) I_{\left(\gamma_{1} \leq 0, \gamma_{2} \leq 0\right)}+h\left(\eta_{1, t}\right) I_{\left(\gamma_{1} \leq 0, \gamma_{2}>0\right)}+h\left(\eta_{1, t}\right) I_{\left(\gamma_{1}>0, \gamma_{2} \leq 0\right)}+h\left(\eta_{1, t}\right) I_{\left(\gamma_{1}>0, \gamma_{2}>0\right)}\right\}\right)^{-1}
$$

with $h\left(\eta_{1, t}\right)$ given in Eqs. (3) and (4).

When $k=2$ and $c_{1} \neq c_{2}$, the model in Eq. (1) nests the following nonlinear model with second order generalized logistic function

$$
G\left(\tilde{\gamma}, h\left(\eta_{t}\right)\right)=1-\exp \left\{-h\left(\eta_{2, t}\right)\right\},
$$

where

$$
h\left(\eta_{2, t}\right)=\left\{\begin{array}{ll}
\gamma_{1}^{-1} \exp \left(\gamma_{1}\left|\left(s_{t}-c_{1}\right)\left(s_{t}-c_{2}\right)\right|-1\right), & \text { if } \gamma_{1}>0 \\
\left(s_{t}-c_{1}\right)\left(s_{t}-c_{2}\right) & \text { if } \gamma_{1}=0 \\
-\gamma_{1}^{-1} \log \left(1-\gamma_{1}\left|\left(s_{t}-c_{1}\right)\left(s_{t}-c_{2}\right)\right|\right), & \text { if } \gamma_{1}<0
\end{array}\right\}
$$


for $\left(s_{t}-c\right)^{2}>0$ (or, equivalently, $\left.h\left(\eta_{t}\right)>1 / 2\right)$ and

$$
h\left(\eta_{2, t}\right)=\left\{\begin{array}{ll}
-\gamma_{2}^{-1} \exp \left(\gamma_{2}^{-1}\left|\left(s_{t}-c_{1}\right)\left(s_{t}-c_{2}\right)\right|-1\right), & \text { if } \gamma_{2}>0 \\
\left(s_{t}-c_{1}\right)\left(s_{t}-c_{2}\right) & \text { if } \gamma_{2}=0 \\
-\gamma_{2}^{-1} \log \left(1-\gamma_{2}\left|\left(s_{t}-c_{1}\right)\left(s_{t}-c_{2}\right)\right|\right), & \text { if } \gamma_{2}<0
\end{array}\right\},
$$

for $\left(s_{t}-c_{1}\right)\left(s_{t}-c_{2}\right)<0$ (or, equivalently, $\left.h\left(\eta_{2, t}\right)<1 / 2\right)$, with $\eta_{t} \equiv \eta_{t}=\left(s_{t}-c_{1}\right)\left(s_{t}-c_{2}\right)$.

A particular case of GSTAR holds when $k=2$ and $c_{1}=c_{2}$, in which case model (1) nests a generalized exponentialautoregressive model (GESTAR). This model is defined in Eqs. (7)-(9), but has $h\left(\eta_{2, t}\right)=\left(s_{t}-c\right)^{2}$ when $\gamma_{1}=0$ for $\left(s_{t}-c\right)^{2}>0$ and $\gamma_{2}=0$ for $\left(s_{t}-c\right)^{2} \leq 0$. In this case, the parameters $\phi+\theta G(\cdot)$ change asymmetrically at some undefined point where the function reaches its own minimum.

The GSTAR nests several well known linear and non-linear models. Before considering the estimation procedure of the GSTAR it is of interest at this point to relate the proposed model to other models available in the literature.

First, model (1) with the transition function in Eq. (5) implies that the GSTAR model reduces to a one-parameter symmetric logistic STAR model (see Teräsvirta (1994)) with slope $\gamma_{1}=\gamma_{2}=\gamma$. However, with respect to the STAR model a clear advantage of the indicator functions in Eq. (5) is that slope parameters are not constrained. Positiveness of the slope parameter is an identifying condition which was a crucial assumption in Teräsvirta (1994). Second, the transition function in Eq. (5) nests an indicator function $I_{\left(s_{t}>c\right)}$ when $\tilde{\gamma} \rightarrow+\infty$. Therefore, the GSTAR reduces to the model in Tong (1983) when $\tilde{\gamma} \rightarrow+\infty$ and it becomes a straight line around $1 / 2$ for each $s_{t}$ when $\tilde{\gamma} \rightarrow-\infty$. Finally, the GSTAR model nests a linear AR model when $\tilde{\gamma}$ is a null vector. In this case $h\left(\eta_{t}\right)$ in Eq. (5) reduces to a simpler function given by

$$
\tilde{h}\left(\eta_{t}\right)=\left\{\begin{array}{ll}
\gamma_{1}^{-1} \exp \left(\gamma_{1}\left|\eta_{t}\right|-1\right) & \text { if } \gamma_{1}>0 \\
0 & \text { if } \gamma_{1}=0 \\
\gamma_{1}^{-1} \log \left(1-\gamma_{1}\left|\eta_{t}\right|\right) & \text { if } \gamma_{1}<0
\end{array}\right\}
$$

for $\eta_{t} \geq 0(\mu>1 / 2)$ and

$$
\tilde{h}\left(\eta_{t}\right)=\left\{\begin{array}{ll}
\gamma_{2}^{-1} \exp \left(\gamma_{2}\left|\eta_{t}\right|-1\right) & \text { if } \gamma_{2}>0 \\
0 & \text { if } \gamma_{2}=0 \\
\gamma_{2}^{-1} \log \left(1-\gamma_{2}\left|\eta_{t}\right|\right) & \text { if } \gamma_{2}<0
\end{array}\right\}
$$

for $\eta_{t}<0(\mu<1 / 2)$. This special case is used below to construct a test for the null of linearity against the alternative hypothesis of dynamic asymmetry.

\subsection{Modelling strategy}

Following standard practice in nonlinear modelling literature, the first step in the model specification procedure is to test whether a linear $A R(p)$ representation is adequate for the data at hand. If the answer is negative, then the second step involves the selection of a nonlinear symmetric model. Crucially, the next step is to test for dynamic symmetry in the house price series. The resulting general-to-specific type of modelling strategy is summarized below:

Step 1. Estimate a suitable linear autoregressive model.

Step 2. Test for the linearity of the model.

Step 3. If the linearity hypothesis is rejected, test the symmetry of the tails of the transition function.

Step 4. If the hypothesis of symmetry is rejected, estimate the GSTAR model.

In Step 1 the correct autoregressive order (say $p$ ) is selected using for example Bayesian Information Criterion and the Portmanteau test for serial correlation. In Step 2 linearity can be tested using the inference procedure suggested in Luukkonen et al. (1988). As far as Step 3 is concerned, the dynamic symmetry hypothesis is tested using a LM-type test. The test statistic is described in detail below. Finally, in Step 4 the choice of the transition function can either be guided by economic theory or alternatively a procedure suggested in Tsay (1989) (see also Teräsvirta (1994)) can be used.

\subsection{Testing for dynamic symmetry}

Testing for linearity against dynamic symmetry is problematic as the GSTAR model is only identified under the alternative hypothesis. In order to overcome this identification problem, following an idea in Luukkonen et al. (1988), we linearize the transition function by taking the third order Taylor expansion of $G(\cdot)$. This approximation leads to an augmented artificial model that can be used to calculate an LM test statistic. 
Consider Eq. (1) with $G\left(\tilde{\gamma}, \tilde{h}\left(\eta_{t}\right)\right) \mid \boldsymbol{\gamma}=0$ and define $\tau=\left(\tau_{1}, \tau_{2}\right)^{\prime}$, where $\tau_{1}=\left(\phi_{0}, \phi^{\prime}\right)^{\prime}, \tau_{2}=\gamma$. Let $\hat{\tau}_{1}$ be the LS estimator of $\tau_{1}$ under $\mathcal{H}_{0}: \tilde{\gamma}=0, \hat{\tau}=\left(\tau_{1}^{\prime}, 0^{\prime}\right)^{\prime}$. Moreover, let $\Xi=[\phi, \theta, \tilde{\gamma}, c], z_{t}(\tau)=\frac{\partial \epsilon_{t}}{\partial \tau}$ and $\hat{z}_{t}=z_{t}(\hat{\tau})=\left(\hat{z}_{1, t}, \hat{z}_{2, t}\right)$, where the partition conforms to that of $\tau$. Then the general form of the LM statistic is

$$
L M(\Xi)=\frac{1}{\hat{\sigma}^{2}} \hat{u}^{\prime} \hat{Z}_{2}\left(Z_{2}^{\prime} \hat{Z}_{2}-\hat{Z}_{2}^{\prime} \hat{Z}_{1}\left(\hat{Z}_{1}^{\prime} \hat{Z}_{1}\right)^{-1} \hat{Z}_{1}^{\prime} \hat{Z}_{2}\right)^{-1} \hat{Z}_{2}^{\prime} \hat{\mathrm{u}}
$$

where $\hat{\mathrm{u}}_{t}=\left[\hat{\mathrm{u}}_{1}, \ldots, \hat{\mathrm{u}}_{T}\right]^{\prime}$ is as previously defined, $\hat{\sigma}^{2}=\frac{1}{T} \sum_{1}^{T} \hat{\mathrm{u}}_{t}^{2}$ and $\hat{\mathrm{u}}_{t}=y_{t}-\hat{\tau}^{\prime} z_{t}, \hat{Z}_{i}=\left(\hat{z}_{i 1}, \ldots, \hat{z}_{i t}, \ldots, \hat{z}_{i T}\right)^{\prime}, i=\{1,2\}$, $t=1, \ldots, T$.

The linearized GSTAR model is given by

$$
y_{t}=\phi^{\prime} z_{t}+\theta^{\prime} z_{t} T_{3}\left[h\left(\eta_{k, t}\right) I_{\left(\gamma_{1} \leq 0, \gamma_{2} \leq 0\right)}+h\left(\eta_{k, t}\right) I_{\left(\gamma_{1} \leq 0, \gamma_{2}>0\right)}+h\left(\eta_{k, t}\right) I_{\left(\gamma_{1}>0, \gamma_{2} \leq 0\right)}+h\left(\eta_{k, t}\right) I_{\left(\gamma_{1}>0, \gamma_{2}>0\right)}+\epsilon_{t}^{\prime}\right] .
$$

The formulation in Eq. (12) leads to the following auxiliary regression for testing linearity and symmetry

$$
\hat{\mathrm{u}}_{t}=\hat{z}_{1 t}^{\prime} \tilde{\beta}_{1}+\sum_{j=1}^{p} \beta_{2 j} y_{t-j} y_{t-d}+\sum_{j=1}^{p} \beta_{3 j} y_{t-j} y_{t-d}^{2}+\sum_{j=1}^{p} \beta_{4 j} y_{t-j} y_{t-d}^{3}+v_{t},
$$

where $v_{t} \sim$ I.I.D. $\left(0, \sigma^{2}\right), \tilde{\beta}_{1}=\left(\beta_{10}, \beta_{1}^{\prime}\right)^{\prime}, \beta_{10}=\phi_{0}-(c / 4) \theta_{0}, \beta_{1}=\phi-(c / 4) \theta+(1 / 4) \theta_{0} e_{d}, e_{d}=(0,0, \ldots, 0,1,0, \ldots, 0)^{\prime}$ with the $d$-th element equal to unit and $T_{3}(G)=f_{1} G+f_{3} G^{3}$ is the third-order Taylor expansion of $G(\Xi)$ at $\tilde{\gamma}=0, f_{1}=\partial G(\Xi) /\left.\partial \Xi\right|_{\gamma=0}$ and $f_{3}=(1 / 6) \partial^{3} G(\Xi) /\left.\partial \Xi\right|_{\gamma=0}, G(\Xi)$ being defined in the previous section. To test the null hypothesis

$$
\mathcal{H}_{0}: \beta_{2 j}=\beta_{3 j}=\beta_{4 j}=0 \quad(j=1, \ldots, p) .
$$

in Eq. (13) the following test statistic can be used

$$
L M=\left(S S R_{0}-S S R\right) / \hat{\sigma}_{v}^{2},
$$

where $S S R_{0}$ and SSR denote the sum of squared estimated residuals from the estimated auxiliary regression (Eq. (12)) and under the null and alternative, respectively, and $\sigma_{v}^{2}=(1 / T) S S R$. Under the null hypothesis the LM test in Eq. (15) is asymptotically distributed as $\chi_{p}^{2}$ distribution.

In a related working paper we investigate the sensitivity of the test statistic to variations of the parameter space and the nonnormality of the innovations by Monte Carlo simulation experiment (see Appendix 2 in Canepa and Chini (2015)). It is found that the inference procedure has good size and power properties for $T>100$. Also, simulation results reveals that the test is relatively well behaved when the innovations are fat-tailed.

\subsection{Estimation}

As far as the estimation of the GSTAR model is concerned, following standard practice (see for example Leybourne et al. (1998)), estimation is performed by concentrating the sum of square residuals function with respect to the vectors $\theta$ and $\phi$, that is minimizing:

$$
\operatorname{SSR}=\sum_{t=1}^{T}\left(y_{t}-\hat{\psi}^{\prime} x_{t}^{\prime}\right)^{2}
$$

where

$$
\hat{\psi}=[\hat{\phi}, \hat{\theta}]=\left(\sum_{t=1}^{T} x_{t}^{\prime}(\tilde{\gamma}, c) x_{t}(\gamma, c)\right)^{-1}\left(\sum_{t=1}^{T} x_{t}^{\prime}(\tilde{\gamma}, c) y_{t}\right),
$$

and

$$
x_{t}(\hat{\gamma}, \hat{c})=\left[z_{t} z_{t}^{\prime} G\left(\hat{\gamma}, h\left(\hat{c}, s_{t}\right)\right)\right]
$$

Note that under the assumption that the vectors $\tilde{\gamma}$ and $c$ are known and fixed, the GSTAR model is linear in the vectors $\theta$ and $\phi$. Therefore, the nonlinear least square minimization problem reduces to a minimization on three (or four) parameters and can be solved via a grid search over $\gamma_{1}, \gamma_{2}$ and $c$. In our application, both $\gamma_{1}$ and $\gamma_{2}$ are chosen between a minimum value of -10 and a maximum of 10 with an increase rate of 0.5 ; whereas the grid for the parameter $c$ is the set the values computed for the range 
of the $10^{\text {th }}$ and $90^{\text {th }}$ percentile of $s_{t}$ with the increase rate computed as the difference of the two percentiles at the boundary divided by an arbitrarily high integer.

Before the estimated GSTAR model can be accepted as adequate, it should be subjected to misspefication tests. Some important hypotheses which should be tested are the hypothesis that there is no residual correlation, no remaining nonlinearity and parameter constancy. These inference procedures are derived in the Appendix.

\section{Data and estimation results}

The data under consideration are obtained from the Bank for International Settlements and relate to quarterly nominal residential properties prices over the period 1970:1 to 2014:1 for the U.S., Spain, UK and Ireland.

The modelling procedure adopted follows the steps described in Section 2. First the maximal lag order of the AR model is chosen by using the Bayesian information criterion and the Portmanteau test for serial correlation. Then, the linearity tests are conducted. To test for linearity the procedure suggested by Luukkonen et al. (1988) is used. This involves testing for the fact that the nonlinear function $G(\cdot)$ is zero in Eq. (6). $\mathcal{H}_{0}: \tilde{\gamma}=0$ against $\mathcal{H}_{1}: \tilde{\gamma} \neq 0$. Under the null the LM-type test is asymptotically distributed as a $\chi^{2}(3 p)$ distribution.

The top panel of Table 1 reports the $p$-values of the test for linearity and the test for dynamic symmetry, respectively. In Table 1 the null hypothesis is rejected at 5\% significance level for U.S., UK and Ireland and $10 \%$ for Spain. Coming to the test

Table 1

Estimated parameters for the GSTAR model and diagnostic tests.

\begin{tabular}{|c|c|c|c|c|}
\hline & U.S. & UK & Ireland & Spain \\
\hline \multicolumn{5}{|l|}{ Linearity and asymmetry tests ( $p$-values) } \\
\hline Test for nonlinearity & 0.031 & 0.023 & 0.002 & 0.072 \\
\hline Test for dynamic symmetry & 0.022 & 0.032 & 0.000 & 0.001 \\
\hline \multicolumn{5}{|l|}{ Estimated parameters } \\
\hline \multirow[t]{2}{*}{$\phi_{0}$} & -0.463 & -0.191 & -6.756 & -4.417 \\
\hline & $0.708)$ & $(2.103)$ & $(4.203)$ & $(10.078)$ \\
\hline \multirow[t]{2}{*}{$\phi_{1}$} & $2.012 * *$ & $1.321 *$ & $1.872 *$ & $-1.433 *$ \\
\hline & $(-1.041)$ & $(0.268)$ & $(0.359)$ & $(0.205)$ \\
\hline \multirow[t]{2}{*}{$\phi_{2}$} & $-1.041 *$ & -0.405 & $-1.288 *$ & $-0.381 * *$ \\
\hline & $(0.335)$ & $(0.307)$ & $(0.5213)$ & $(0.218)$ \\
\hline \multirow[t]{2}{*}{$\theta_{10}$} & $1.823 *$ & $3.433 *$ & $7.997 * *$ & $17.57 * *$ \\
\hline & $(0.730)$ & $(0.214)$ & $(4.209)$ & $(10.108)$ \\
\hline \multirow[t]{2}{*}{$\theta_{11}$} & $-0.974 *$ & 0.349 & $-0.973 *$ & $-0.488 *$ \\
\hline & $(0.366)$ & $(0.272)$ & $(0.360)$ & $(0.220)$ \\
\hline \multirow[t]{2}{*}{$\theta_{12}$} & $0.869 *$ & -0.457 & $1.273 *$ & 0.324 \\
\hline & $(0.335)$ & $(0.309)$ & $(0.522)$ & $(0.246)$ \\
\hline \multirow[t]{2}{*}{$\gamma_{1}$} & $9.000 *$ & $4.800 *$ & 3.600 & $5.000 *$ \\
\hline & $(0.491)$ & $(2.214)$ & $(4.209)$ & $(0.417)$ \\
\hline \multirow[t]{2}{*}{$\gamma_{2}$} & $-9.000 *$ & $-4.800 *$ & $-3.600 *$ & $-5.000 *$ \\
\hline & $(0.480)$ & $(0.272)$ & $(0.360)$ & $(0.388)$ \\
\hline \multirow[t]{2}{*}{$c$} & $6.659 *$ & $4.268 *$ & $-4.330 *$ & $19.895 *$ \\
\hline & $(0.094)$ & $(0.309)$ & $(0.553)$ & $(0.358)$ \\
\hline \multicolumn{5}{|l|}{ Diagnostic tests ( $p$-values) } \\
\hline \multicolumn{5}{|l|}{ LM test for no error correlation } \\
\hline$q=1$ & 0.799 & 0.858 & 0.962 & 0.381 \\
\hline$q=2$ & 0.968 & 0.984 & 0.998 & 0.682 \\
\hline$q=4$ & 0.999 & 0.999 & 0.999 & 0.944 \\
\hline \multicolumn{5}{|l|}{ F test for no error correlation } \\
\hline$q=1$ & 0.800 & 0.513 & 0.922 & 0.380 \\
\hline$q=2$ & 0.893 & 0.808 & 0.997 & 0.680 \\
\hline$q=4$ & 0.999 & 0.980 & 1.000 & 0.937 \\
\hline LM test for no remaining asymmetry & 0.999 & 0.998 & 0.999 & 0.998 \\
\hline \multicolumn{5}{|l|}{ LM test for parameter constancy } \\
\hline $\mathrm{H} 1$ & 0.999 & 0.999 & 0.999 & 0.999 \\
\hline $\mathrm{H} 2$ & 0.999 & 0.995 & 0.999 & 0.998 \\
\hline H3 & 0.999 & 0.989 & 0.999 & 0.999 \\
\hline
\end{tabular}

The table reports the linearity and dynamic symmetry tests in the top panel. The estimated parameters are reported in the middle panel and $p$-values for the misspecification tests are given in the bottom panel. The diagnostic statistics are: $i$ ) the LM and the F tests for the hypothesis that there is no serial correlation against the $q$-order autoregression, $\mathrm{ii}$ ) the LM test for the hypothesis that there is no remaining asymmetry, iii) the LM test for parameter constancy. Note:

* Indicates significance level at $5 \%$

** Indicates significance level at $10 \%$. 
for dynamic symmetry, the LM-type test statistic hasunder the null hypothesis $\mathcal{H}_{0}: \tilde{\gamma}=0, \hat{\tau}=\left(\tau_{1}^{\prime}, 0^{\prime}\right)^{\prime}$ which, as seen in the previous section, corresponds to testing the null hypothesis

$$
\mathcal{H}_{0}: \beta_{2 j}=\beta_{3 j}=\beta_{4 j}=0 \text {, for } j=1, \ldots, p,
$$

in the auxiliary Eq. (12). From Table 1, on the basis of the empirical p-values reported in column three, the null hypothesis of dynamic symmetry can be rejected.

With regard to the transition function, as discussed in Section 2, the choice of the number of location parameters $k$ affects the type of asymmetric behaviour characterized by the model. The expression in Eq. (6) with $k=1$ is suitable to describe housing markets with a rapid change in house prices during expansions but with a slow rate of change during recessions. On the other hand, when $k=2$ in Eq. (6), the resulting exponential form of the transition function suggests that recessions and recovery phases have similar dynamics but different durations and intensities. This feature is referred to as "deepness" of the cycle in Sichel (1993). The GSTAR model introduced in Section 2 is capable of describing both types of asymmetric behavior in house price changes. However, in this context we are more interested in modelling situations where expansions and recessions have different dynamic regimes rather than midrange regimes. For this reason, the model in Eq. (6) with $k=1$ is considered.

In Table 1 the estimated parameters are reported. From Table 1 it appears that most estimated $\theta$ coefficients for the house prices under consideration are significantly different from zero. The parameters $\gamma_{1}$ and $\gamma_{2}$ indicate the speed of the transition between regimes. These coefficients are also significantly different from zero. With regard to the signs of these coefficients it is observed that the parameter $\gamma_{1}$ is always positive, whereas $\gamma_{2}$ is negative, regardless the country taken into consideration. This indicates that the speed of the transition from one regime to the other regime increases during periods of house price booms at a rate that is greater than one which would be consistent with a standard logistic curve, but decreases during the periods of house price recessions at a rate which is slower than one that would be consistent with a standard logistic function. The signs of the estimated $\gamma_{1}$ and $\gamma_{2}$ are consistent with asymmetric price adjustment models found in the applied economic literature. The fact that house price changes deviate above their mean at an exponential rate, whereas they return at a logarithmic rate implies that when improving economic conditions boost the housing demand above the potential stock, prices raise rapidly above their expected level, but they fall slowly when economic conditions worsen and price changes are below the expect value. This suggests that for a given housing stock, a positive demand shock pushes prices up, but it will have a relatively small effect on housing supply. In contrast, a negative demand shock will have a relatively large impact on housing supply.

In relation to the magnitude of the estimated $\gamma_{1}$ and $\gamma_{2}$ coefficients, ${ }^{1}$ these parameters take values 9.0 for the U.S., approximately 5.0 for the UK and Spain and 3.6 for Ireland. The greater magnitude of the speed parameters $\gamma_{1}$ and $\gamma_{2}$ indicates a sharper transition for the U.S. from one regime to another in this country with respect to the European countries. This is probably due to the more conservative housing policies in Europe. Note that the relatively small estimates of $\gamma_{1}$ and $\gamma_{2}$ indicate that other types of nonlinear models in the class of regime switching, such as the Markov switching or the TAR models, are no suitable to capture housing market dynamics since these models assume a sudden transition between one regime and the other (i.e. in these models $\gamma_{1}=\gamma_{2} \rightarrow \infty$ by assumption). Coming now to the parameter $c$, this indicates the halfway point between the expansion and contraction phases of the housing markets. In all cases, the values of $c$ is statistically significant at the $5 \%$ level. The estimated parameters $c$ are positive for all countries, but Ireland.

Once that the model has been estimated, its goodness of fit can be evaluated using misspecification tests. The diagnostic statistics considered here are: $i$ ) the LM and the F tests for the hypothesis that there is no serial correlation against the $q$-order autoregression (for $q \in\{1, \ldots, 4\}$ ), ii) the LM test the hypothesis that there is no remaining asymmetry, iii) the LM test for parameter constancy. The misspecification tests are derived in Appendix.

The $p$-values of the tests are reported in the bottom panel of Table 1. Looking at the results of the misspecification tests it emerges that the LM and the F tests do not reject the null hypotheses of no autocorrelation against $q$-order autoregression for all estimated models. There is also no evidence of remaining asymmetry given that the LM test does not reject the null hypothesis for the estimated models. Similarly, the LM test of parameter constancy also does not reject the null hypothesis at the 5\% significant level for all the estimated models. Overall, the results in Table 1 suggest that the estimated models do not suffer from misspecification problems.

Fig. 1 reports the estimated transition functions for the housing markets under consideration plotted against the transition variable $\left(s_{t}\right)$ with one dot for every observation (note that a single dot may represent more than one observation). The plot of the estimated transition functions shed further light on the degree of asymmetry of the sigmoid function. For the UK housing market almost two thirds of the observations lie in the lower part of the graph, corresponding to the segment between 0.2 and 0.4 of the vertical axis, while the remaining are in the extreme regime. The plot of the estimated transition of the Spanish housing market is similar to that of the UK market. However, the transition function of the U.S. shows more more extreme behaviour, as confirmed by the greater magnitude of the nonlinear parameters. On the other hand, in the case of the Irish housing market these proportions are inverted. In Fig. 1, the shape of the transition functions reflect the different signs of the estimated slope parameters in Table 1: the negative sign of $\gamma_{2}$ corresponds to a logarithmic rescaling of the segment $0-0.5$ of the vertical axis, whereas the positive $\gamma_{1}$ produces an exponential transformation of the upper part.

\footnotetext{
${ }^{1}$ Note that the magnitudes of the two slopes should not be confused with symmetry, in that this magnitude simply indicates that a strong departure from the conditional mean is followed by a slow recovery.
} 


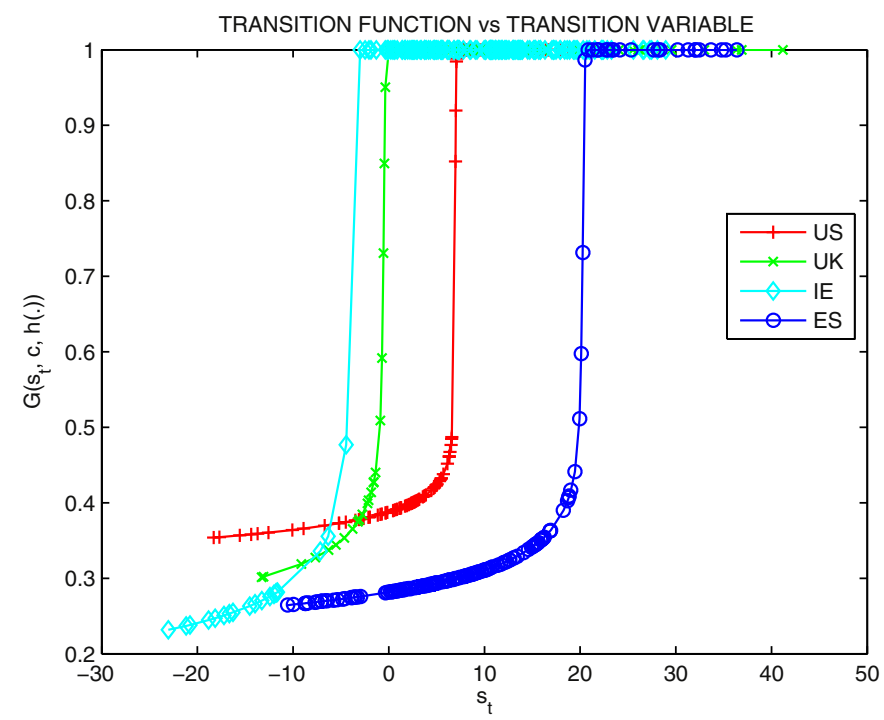

Fig. 1. Estimated transition functions and the transition variables. Note: each dot represents at least one observation.

The estimated transition functions are shown over time in Fig. 2 along with house price changes, $\Delta y_{\text {t. }}$ In Fig. 2 the transition functions for the estimated models take values at or close to one during periods of price booms and values close to zero during periods of housing market busts. Overall, looking at the plots of the two regimes it emerges that the GSTAR model captures reasonably well contractions and expansions in the housing markets under consideration.
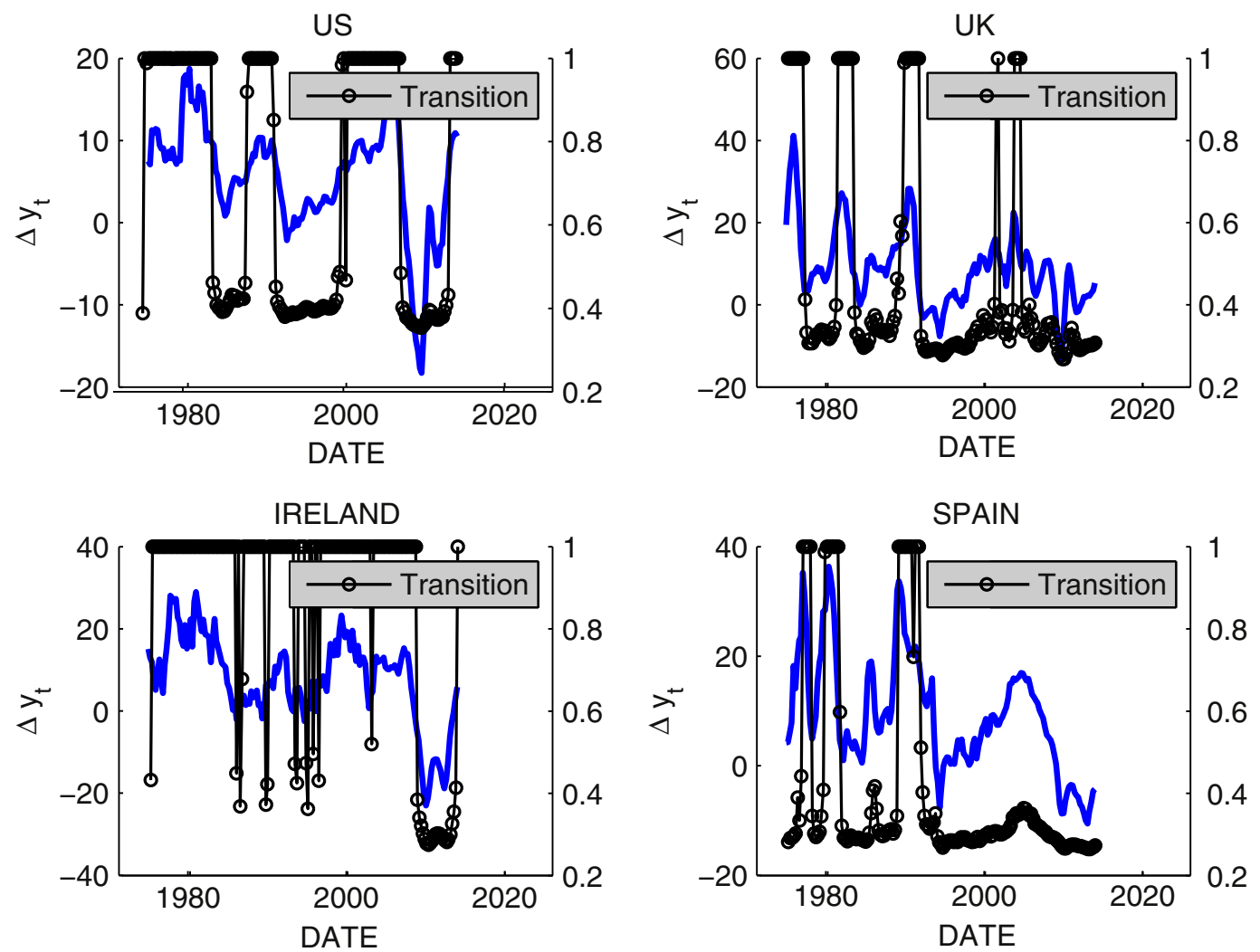

Fig. 2. Observed values and estimated transition functions over time. 


\subsection{Forecasting house prices}

A rolling forecast experiment is implemented in order to investigate the forecasting ability of the GSTAR model.

With this target in mind the house price series are split onto two subsamples: a pre-forecast period (for $\left.t=1, \ldots, T_{s-1}\right)$ from which the model is estimated and a forecast period $t=T^{s}, \ldots, T$ with $T^{s}=t+h$. Then $h$-step-ahead forecasts are computed and compared with the pre-forecast period. The forecast period under consideration is $h=\{1,3,6,12\}$.

For each country we compare a linear AR(3) model and the GSTAR model in their out-of-sample point forecasts. The out-ofsample forecast comparisons do not rely on a single criterion, for robustness we compare the results of four different measures. Namely, the mean forecast error (MFE), the root mean square forecast error (RMSFE), the symmetric mean absolute percentage error (SMAPE) and the median relative absolute error (mRAE). The four performance measures are calculated as follows:

$$
\begin{aligned}
& M F E_{h}=\frac{1}{T-h-T^{s}+1} \sum_{t=T^{s}}^{T-h}\left(\Delta y_{t+h}-\Delta \hat{y}_{t+h \mid t}\right), \\
& S M A P E_{h}=\frac{100\left|\Delta y_{t+h}-\Delta \hat{y}_{t+h}\right|}{0.5\left(\Delta y_{t+h}-\Delta \hat{y}_{t+h \mid t}^{j}\right)}, \\
& m R A E_{h}=\frac{\left|\Delta y_{t+h}-\Delta \hat{y}_{t+h}\right|}{\left|\Delta y_{t+h}-\Delta \hat{y}_{t+h}^{(1)}\right|}, \text { with Eq. (1) indexing the benchmark model; } \\
& \operatorname{RMSFE}_{h}=\frac{1}{T-h-T^{s}+1} \sum_{t=T^{s}}^{T-h}\left(\Delta y_{t+h}-\Delta \hat{y}_{t+h \mid t}\right)^{2} .
\end{aligned}
$$

Table 2 reports the results of the forecasting exercise. In columns 2 and 3 the forecasting horizon and the forecast error measures are reported, respectively, whereas in columns 4-7 the forecasting results for each housing market are reported.

\begin{tabular}{|c|c|c|c|c|c|c|}
\hline Model & Forecast horizon & Forecast error measure & U.S. & UK & Ireland & Spain \\
\hline \multirow[t]{4}{*}{$A R(3)$} & 1 & \multirow[t]{4}{*}{ MFE } & 0.0137 & -0.177 & -0.022 & -0.536 \\
\hline & 3 & & 0.2537 & -0.195 & -0.050 & -0.535 \\
\hline & 6 & & 0.4541 & -0.206 & -0.077 & -0.571 \\
\hline & 12 & & 0.5757 & -0211 & -0.223 & -0.594 \\
\hline \multirow[t]{4}{*}{ GSTAR } & 1 & \multirow[t]{4}{*}{ MFE } & -0.073 & -0.130 & -0.148 & -0.166 \\
\hline & 3 & & -0.069 & -0.195 & -0.163 & -0.178 \\
\hline & 6 & & -0.078 & -0.207 & -0.173 & -0.143 \\
\hline & 12 & & -0.113 & -0.208 & -0.246 & -0.138 \\
\hline \multirow[t]{4}{*}{ AR } & 1 & \multirow[t]{4}{*}{ sMAE } & 0.004 & 0.003 & 0.006 & 0.004 \\
\hline & 3 & & 0.005 & 0.003 & 0.007 & 0.004 \\
\hline & 6 & & 0.006 & 0.004 & 0.007 & 0.004 \\
\hline & 12 & & 0.008 & 0.004 & 0.006 & 0.004 \\
\hline \multirow[t]{4}{*}{ GSTAR } & 1 & \multirow[t]{4}{*}{ SMAE } & 0.003 & 0.004 & 0.003 & 0.004 \\
\hline & 3 & & 0.003 & 0.005 & 0.00 & 0.004 \\
\hline & 6 & & 0.003 & 0.007 & 0.005 & 0.004 \\
\hline & 12 & & 0.004 & 0.003 & 0.005 & 0.004 \\
\hline \multirow[t]{4}{*}{ AR } & 1 & \multirow[t]{4}{*}{ mRAE } & 1.000 & 1.000 & 1.000 & 1.000 \\
\hline & 3 & & 1.000 & 1.000 & 1.000 & 1.000 \\
\hline & 6 & & 1.000 & 1.000 & 1.000 & 1.000 \\
\hline & 12 & & 1.000 & 1.000 & 1.000 & 1.000 \\
\hline \multirow[t]{4}{*}{ GSTAR } & 1 & \multirow[t]{4}{*}{ mRAE } & 1.257 & 0.414 & 1.071 & 1.046 \\
\hline & 3 & & 1.481 & 0.424 & 1.199 & 1.075 \\
\hline & 6 & & 1.924 & 0.406 & 1.480 & 1.032 \\
\hline & 12 & & 2.174 & 0.733 & 1.334 & 1.95 \\
\hline \multirow[t]{4}{*}{$\operatorname{AR}(3)$} & 1 & \multirow[t]{4}{*}{ RMSFE } & 0.197 & 0.315 & 0.329 & 0.226 \\
\hline & 3 & & 0.202 & 0.317 & 0.331 & 0.228 \\
\hline & 6 & & 0.220 & 0.421 & 0.339 & 0.233 \\
\hline & 12 & & 0.254 & 0.932 & 0.346 & 0.242 \\
\hline \multirow[t]{4}{*}{ GSTAR } & 1 & \multirow[t]{4}{*}{ RMSFE } & 0.140 & 0.286 & 0.280 & 0.226 \\
\hline & 3 & & 0.142 & 0.305 & 0.284 & 0.228 \\
\hline & 6 & & 0.145 & 0.406 & 0.290 & 0.231 \\
\hline & 12 & & 0.150 & 0.920 & 0.302 & 0.242 \\
\hline
\end{tabular}
From the top panel of Table 2 it is clear that according to the MFE and sMAE criteria the GSTAR model performs better than its

Table 2

Forecasting house prices: point predictive performances.

The table compares for each country a linear AR(3) model and the GSTAR model in their out-of-sample point forecasts. The forecast measures are $i$ ) the mean forecast error (MFE), ii) the root mean square forecast error (RMSFE), iii) the symmetric mean absolute percentage error (sMAPE), $i v$ ) the median relative absolute error (mRAE). The forecast horizon is $1,3,6$, and 12 quarters. 
symmetric counterpart. Similarly, in the bottom panel, according to the RMSFE the GSTAR has superior forecasting properties. However, looking at the mRAE measure the results are mixed.

\section{Conclusion}

In this paper we introduce a novel nonlinear model to capture dynamic asymmetries in house price cycles. The suggested model is capable of parametrizing the asymmetry in the transition equation by using a particular generalization of the logistic function. Although applied in this paper specifically to the analysis of housing markets, the proposed model can be used to test for dynamic asymmetries in a more general context. The application to house prices reveals several insights into the patterns of the housing markets under consideration. It is found that house prices increase at exponential rate during expansion periods, whereas contractions in home prices follow decrease at a logarithmic rate. Therefore, contractions occur for a more prolonged period than expansions. An interesting conclusion from our analysis is that the direction of the estimated asymmetry is the same for all countries considered.

In the aftermath of the global financial crisis, there is a growing consensus that the housing sector plays a key role in financial stability. The recent financial crisis started in the housing sector and escalated further in the financial sector and before long the ensuing dip recession hit the real economy causing a high level of unemployment and spreading misery throughout the world. Good prudential policy requires action before the housing sector overheats. Hence our findings regarding the asymmetric behaviour of house prices over the business cycles may be useful to policy makers and could, perhaps, help to encourage their willingness to lean against the wind.

\section{Appendix A. Misspecification tests}

Like any other model, the GSTAR needs to be evaluated for possible misspecification before it can be used for forecasting purposes. To investigate the quality of the estimated model, the tests for serial independence, no remaining asymmetry and parameter constancy are derived below. The suggested inference procedures are derived as a generalization of the misspecification tests in Eitrheim and Teräsvirta (1996).

\section{A.1. Test for serial independence}

Consider the general additive model in Eq. (1), where

$$
\epsilon_{t}=a^{\prime} v_{t}+u_{t}=\sum_{j=1}^{q} a_{j} L^{j} \epsilon_{t}+u_{t}, \quad u_{t} \sim \text { I.I.D. }\left(0, \sigma^{2}\right),
$$

with $L^{j}$ denoting the lag operator, $v_{t}=\left(u_{t-1}, \ldots, u_{t-q}\right)^{\prime}, a=\left(a_{1}, \ldots, a_{q}\right)^{\prime}, a_{q} \neq 0$. Under the assumption of stationarity and ergodicity, the null hypothesis of serial independence is $\mathcal{H}_{0}: a=0$. By pre-multiplying Eq. (2) by $1-\sum_{j=1}^{q} a_{j} L^{j}$ we obtain

$$
y_{t}=\sum_{j} a_{j} L^{j} y_{t}+\phi^{\prime} z_{\mathbf{t}}-\sum_{j} a_{j} L^{j} \phi^{\prime} z_{t}+\theta^{\prime} z_{t} G(\cdot)-\sum_{j} a_{j} \theta^{\prime} G(\cdot)+\epsilon_{t} .
$$

Hence, assuming that the initial values $y_{0}, y_{-1}, \ldots, y_{-(p+q)+1}$ are fixed, the pseudo normal likelihood for $t=1, \ldots, T$ is

$$
\begin{aligned}
\mathcal{L}_{t} & =\text { constant }+\frac{1}{2} \ln \sigma^{2}-\frac{\epsilon_{t}^{2}}{2 \sigma^{2}}, \\
\epsilon_{t} & =y_{t}-\sum_{j} a_{j} L^{j} y_{t}-\phi^{\prime} z_{t}+\sum_{j} a_{j} L^{j} \phi^{\prime} z_{t}-\theta^{\prime} G\left(z_{t-j}, \exists\right)+\sum_{j} a_{j} \theta^{\prime} G\left(z_{t-j}, \exists\right) .
\end{aligned}
$$

Consistent with initial assumptions of the model, the information matrix is block diagonal and $\sigma^{2}$ is fixed. Therefore, the partial derivatives with respect to $a_{j}$ and $\exists$ are given by

$$
\begin{aligned}
& \frac{\partial \mathcal{L}_{t}}{\partial a_{j}}=\frac{\epsilon_{t}}{\sigma^{2}}\left[y_{t-j}-\phi^{\prime} z_{t-j}-\theta^{\prime} G\left(z_{t-j}, \Xi\right)\right], \\
& \frac{\partial \mathcal{L}_{t}}{\partial \Xi}=\frac{\epsilon_{t}}{\sigma^{2}}\left[\theta^{\prime} z_{t} \frac{\partial G\left(z_{t-j}, \Xi\right)}{\partial \Xi}-\sum_{j} a_{j} \theta^{\prime} \frac{\partial G\left(z_{t-j}, \Xi\right)}{\partial \Xi}\right] .
\end{aligned}
$$

Under the null hypothesis, consistent estimators of Eqs. (3A)-(4A) are given by

$$
\left.\frac{\partial \hat{\mathcal{L}}_{t}}{\partial a_{t}}\right|_{\mathcal{H}_{0}}=\frac{1}{\sigma^{2}} \hat{\mathbf{u}}_{t} \hat{v}_{t} \text { and }\left.\frac{\partial \hat{\mathcal{L}}_{t}}{\partial \Xi}\right|_{\mathcal{H}_{0}}=-\frac{1}{\sigma^{2}} \hat{\mathrm{u}}_{t} \hat{z}_{t}
$$


where the vector $\hat{\mathrm{u}}_{t}=\left(\hat{v}_{t-1}, \ldots, \hat{v}_{t-q}\right)^{\prime}, \hat{v}_{t-j}=y_{t-j}-\phi^{\prime} z_{t-j}-\theta^{\prime} G\left(z_{t-j}, \hat{\Xi}\right), j=1, \ldots, q, \hat{\Xi}$ is the QMLE estimator of $\Xi$ and

$$
\hat{z}_{t}=\frac{\partial G\left(z_{t}, \hat{\Xi}\right)}{\partial \hat{\Xi}}=k_{t}^{G}=\left[\theta^{\prime} z_{t} G_{\gamma 1}, \theta^{\prime} z_{t} G_{\gamma 2}, \theta^{\prime} z_{t} G_{\gamma c}\right] .
$$

The resulting LM test statistic is given by

$$
L M=\frac{1}{\hat{\sigma}}\left(\hat{\mathbf{u}}_{t}^{\prime} \hat{v}_{t}\right)\left\{\hat{v}_{t}^{\prime} \hat{v}_{t}-\hat{v}_{t}^{\prime} \hat{z}_{t}\left(\hat{z}_{t} \hat{z}_{t}^{\prime}\right)^{-1} \hat{z}_{t}^{\prime} \hat{v}_{t}\right\}^{-1}\left(\hat{v}^{\prime} \hat{\mathbf{u}}_{t}\right)
$$

with $\hat{\sigma}^{2}=\frac{1}{T} \sum_{t} u_{t}^{2}$. Under the null hypothesis the statistic Eq.(6A) is asymptotically $\chi_{q}^{2}$ distributed.

Note that in finite and moderate samples the LM statistic can be severely size-distorted. In general, the size distortion of the LM test is sensitive to the number of nuisance parameters. It may also be the case that the estimated residual vector could be non-orthogonal to the gradient vector $\hat{z}_{t}$. A small sample corrected $F$-type test is therefore proposed below. To calculate the test one may proceed as follows:

Step 1. Estimate the GSTAR model under the assumption of uncorrelated errors and compute the residual sum of squares $S S R_{0}=\sum_{t=1}^{T} \hat{\mathrm{u}}_{t}^{2}$.

Step 2. Regress $\hat{\mathrm{u}}_{t}$ on $\hat{v}_{t}, z_{t}, z_{t} \hat{\mathrm{G}}\left(z_{t-d}\right), \hat{G}_{\gamma 1}, \hat{G}_{\gamma 2}, \hat{G}_{c}$ and compute SSR;

Step 3. Compute the test $F$-type statistic

$$
F=\frac{q^{-1}\left(S S R_{0}-S S R\right)}{(T-n-q)^{-1} S S R}, \quad \text { where } \quad n=\operatorname{dim}\left(\hat{\mathbf{z}}_{t}\right)
$$

A variation of the suggested procedure is proposed in Eitrheim and Teräsvirta (1996) where an extra-step is added to Step 2. Eitrheim and Teräsvirta (1996) suggest regressing the estimated errors on $z_{t}, z_{t}, z_{t} \hat{G}\left(z_{t-j}\right), \hat{G}_{\gamma 1}, \hat{G}_{\gamma 2}, \hat{G}_{c}$ and then using the resulting errors $\tilde{u}_{t}$ to calculate $S S R_{1}=\sum_{t=1}^{T} \tilde{u}_{t}^{2}$.

\section{A.2. Test for no remaining asymmetry}

Consider the additive GSTAR model

$$
y_{t}=\phi^{\prime} z_{t}+\theta^{\prime} z_{t} G_{1}\left(\tilde{\gamma}, h\left(\eta_{t}\right)^{(1)}\right)+\pi^{\prime} z_{t} G_{2}\left(\tilde{\gamma}, \tilde{h}\left(\eta_{t}\right)^{(2)}\right)+u_{t}
$$

with $u_{t} \sim$ I.I.D. $\left(0, \sigma^{2}\right)$. The null of neglected asymmetry is

$$
\mathcal{H}_{0}: \tilde{h}\left(\eta_{t}\right)^{(2)}=0 \text { versus } \mathcal{H}_{1}: \tilde{h}\left(\eta_{t}\right)^{(2)} \neq 0
$$

If the vector $\tilde{\gamma}$ is found to be not null, the investigator can easily check if the additive nonlinear part is significant.

Under the null hypothesis we assume that $\Xi$ can be consistently estimated by QML. However, the model in Eq.(7A) is not identified under the null hypothesis. Therefore, once again a Taylor expansion of the transition function $G(\cdot)$ can be used in order to circumvent the problem. In this case, assume that $G_{2}(\cdot)$ is a generalized logistic function and replace it with its third-order Taylor expansion about $h(\gamma)^{(2)}=0$. This implies

$$
T_{2}=g_{20}+g_{21} y_{t-l}+g_{22} y_{t-l}^{2}+g_{23} y_{t-l}^{3}
$$

where $g_{2 j}, j=0,1,2,3,4$ are functions of the vector $\gamma^{(2)}$ such that $g_{20}=g_{21}=g_{22}=g_{23}=0$ for $\gamma^{(2)}=0$, consistent with the definition of $h_{\gamma}\left(s_{t}\right)$. Re-parametrizing the expression in Eq. (7A) the model becomes

$$
y_{t}=\beta_{0}^{\prime} z_{t}+\theta^{\prime} z_{t} G_{1}(\cdot)+\beta_{1}^{\prime} \tilde{z}_{t} y_{t-l}+\beta_{2}^{\prime} \tilde{z}_{\mathbf{t}} y_{t-l}^{2}+\beta_{3}^{\prime} \tilde{z}_{\mathbf{t}} y_{t-l}^{3}+r_{t}
$$

where $\tilde{z}_{t}=\left(y_{t-1}, \ldots, y_{t-p}\right)^{\prime}$. The null hypothesis of no additive nonlinearity is $\mathcal{H}_{0}: \beta_{1}=\beta_{2}=\beta_{3}=0$ (note also that under the null hypothesis $r_{t}=u_{t}$ ). The $L M$ statistic is asymptotically distributed as a $\chi^{2}(3 p)$. Since there are no modifications in the statistical assumptions concerning the error distribution, the asymptotic distribution of the test can be derived as in Eitrheim and Teräsvirta (1996). 


\section{A.3. Test for parameter constancy}

Consider the model

$$
y_{t}=\phi(t)^{\prime} \bar{z}_{t}+\theta(t)^{\prime} \tilde{z}_{t} G\left(\gamma, h\left(\eta_{t}\right)\right)+u_{t}, \quad u_{t} \sim \text { I.I.D. }\left(0, \sigma^{2}\right),
$$

with $\bar{z}_{t}$ denoting the $k \leq p+1$ element of $z_{t}$ for which the corresponding element of $\phi$ is not assumed zero a priori, $\tilde{z}_{\mathbf{t}}$ is the same $(l \times 1)^{\prime}$ for the element of $\theta$. Let $\tilde{\phi}$ and $\tilde{\theta}$ denote the equivalent $(k+1)$ and $(l+1)$ parameter vectors, $\phi(t)=\tilde{\phi}+\lambda_{1} G_{j}\left(t ; \tilde{\gamma}, h\left(\eta_{t}\right)^{(1)}\right)$, and $\theta(t)=\tilde{\theta}+\lambda_{2} G_{j}\left(t ; \tilde{\gamma}, h\left(\eta_{t}\right)^{(2)}\right)$ with $\lambda_{1}$ and $\lambda_{2}$ being $(k \times 1)$ and $(l \times 1)$ vectors, respectively. Then the null hypothesis of parameter constancy in Eq. $(11 \mathrm{~A})$ is

$$
\mathcal{H}_{0}: G\left(t ; \gamma, h\left(\eta_{t}\right)\right) \equiv 0
$$

Three forms for $G(\cdot)$ can be considered

$$
\begin{aligned}
G_{1}\left(t ; \gamma, h\left(c, s_{t}\right)\right) & =\left(1+\exp \left\{-h\left(\eta_{t}^{G L}\right)\right\}\right)^{-1} \quad \text { with } \\
\eta_{t}^{G L} & \equiv t-c, \\
G_{2}\left(t ; \gamma, h\left(c, s_{t}\right)\right) & =\left(1+\exp \left\{-h\left(\eta_{t}^{G E}\right)\right\}\right)^{-1} \quad \text { with } \\
\eta_{t}^{G L} & \equiv(t-c)^{2}, \\
G_{3}\left(t ; \gamma, h\left(c, s_{t}\right)\right) & =\left(1+\exp \left\{-h\left(\eta_{t}^{c}\right)\right\}\right) \quad \text { with } \\
\eta_{t}^{C} & \equiv\left(t^{3}-c_{12} t^{2}+c_{11} t+c_{10}\right) .
\end{aligned}
$$

The null of parameter constancy is $\mathcal{H}_{0}: \tilde{\gamma}=0$. Note that in this case the model is identified also in the case of $\tilde{\gamma}<0$, so that the only identifying restriction is that $\tilde{\gamma} \neq 0 . G_{1}$ and $G_{2}$ are the generalized logistic and the exponential smooth transition of the change in parameters, respectively. The function $G_{3}$ is a cubic function which allows for both monotonically and nonmonotonically changing parameters and can be seen as a general case of $G_{1}$ and $G_{2}$ when constructing the test. As suggested by the literature, we use a third-order Taylor expansion of $G_{3}$ about $\tilde{\gamma}=0$

$$
T_{3}\left(t ; \tilde{\gamma}, h\left(\eta_{t}\right)\right)=\frac{1}{4} h(\tilde{\gamma})\left(t^{3}+c_{12} t^{2}+c_{11} t+c_{10}\right)+R\left(t, \tilde{\gamma}, h\left(\eta_{t}\right)\right) .
$$

in order to approximate $\phi(t)$ and $\theta(t)$ in Eq. (11A) using Eq. (14A). This yields

$$
y_{t}=\beta_{0}^{\prime}\left(\bar{z}_{t}\right)+\beta_{1}^{\prime}\left(t \overline{\mathbf{z}}_{t}\right)+\beta_{2}^{\prime}\left(t^{2} \overline{\mathbf{z}}_{t}\right)+\beta_{3}^{\prime}\left(t^{3} \overline{\mathbf{z}}_{t}\right)+y_{t}+\left\{\beta_{4}^{\prime}\left(\tilde{\mathbf{z}}_{t}\right)+\beta_{5}^{\prime}\left(t \tilde{\mathbf{z}}_{t}\right)+\beta_{6}^{\prime}\left(t^{2} \tilde{\mathbf{z}}_{t}\right)+\beta_{7}^{\prime}\left(t^{3} \tilde{\mathbf{z}}_{t}\right)\right\} G\left(t ; \tilde{\gamma}, h\left(\eta_{t}\right)\right)+r_{t}^{*},
$$

where $r_{t}^{*}=u_{t}+R\left(t ; \tilde{\gamma}, h\left(\eta_{t}\right)\right)$. Under $\mathcal{H}_{0}, r_{t}^{*}=u_{t}$. In Eq. $(11 \mathrm{~A}), \beta_{j}=h\left(\eta_{t}\right) \bar{\beta}, j=1, \ldots, 7$, hence the null hypothesis in terms of Eq. (15A) becomes $\mathcal{H}_{0}: \beta_{j}=0, j=1, \ldots, 7$. Consequently, the locally approximated pseudo normal log-likelihood under $\mathcal{H}_{0}$ (ignoring $R$ ) is

$$
\begin{aligned}
\mathcal{L}_{t}= & \text { const }-\frac{1}{2} \ln \sigma^{2}-\frac{1}{2} \sigma^{2}\left[y_{t}-\beta_{0}^{\prime} w_{t}-\beta_{1}^{\prime}\left(t \bar{w}_{t}\right)-\beta_{2}^{\prime}\left(t^{2} \bar{w}_{t}\right)-\beta_{3}^{\prime}\left(t^{3} \bar{w}_{t}\right)-\right. \\
& -\left\{\beta_{4}^{\prime}\left(\tilde{w}_{t}\right)+\beta_{5}^{\prime}\left(t \tilde{w}_{t}\right)+\beta_{6}^{\prime}\left(t^{2} \tilde{w}_{t}\right)+\beta_{7}^{\prime}\left(t^{3} \tilde{w}_{t}\right)\right\} G\left(y_{t-d} ; \tilde{\gamma}, h\left(\eta_{t}\right)\right]^{2} .
\end{aligned}
$$

The partial derivatives are:

$$
\begin{aligned}
& \frac{\partial \mathcal{L}_{t}}{\partial \beta_{j}}=\frac{1}{\sigma^{2}} u_{t}\left(t^{t} \bar{w}_{t}\right), \quad j=0, \ldots, 3, \\
& \frac{\partial \mathcal{L}_{t}}{\partial \beta_{j}}=\frac{1}{\sigma^{2}} u_{t}\left(t^{j} \tilde{w}_{t}\right) G\left(y_{t-d} ; \tilde{\gamma}, h\left(\eta_{t}\right)\right), \quad j=4, \ldots, 7, \\
& \frac{\partial \mathcal{L}_{t}}{\partial \gamma_{1}}=\frac{1}{\sigma^{2}} u_{t}\left\{\beta_{4}^{\prime}\left(\tilde{w}_{t}\right)+\beta_{5}^{\prime}\left(t \tilde{w}_{t}\right)+\beta_{6}^{\prime}\left(t^{2} \tilde{w}_{t}\right)+\beta_{7}^{\prime}\left(t^{3} \tilde{w}_{t}\right)\right\} G_{\gamma_{1}}, \\
& \frac{\partial \mathcal{L}_{t}}{\partial \gamma_{2}}=\frac{1}{\sigma^{2}} u_{t}\left\{\beta_{4}^{\prime}\left(\tilde{w}_{t}\right)+\beta_{5}^{\prime}\left(t \tilde{w}_{t}\right)+\beta_{6}^{\prime}\left(t^{2} \tilde{w}_{t}\right)+\beta_{7}^{\prime}\left(t^{3} \tilde{w}_{t}\right)\right\} G_{\gamma_{2}},
\end{aligned}
$$




$$
\frac{\partial \mathcal{L}_{t}}{\partial c}=\frac{1}{\sigma^{2}} u_{t}\left\{\beta_{4}^{\prime}\left(\tilde{w}_{t}\right)+\beta_{5}^{\prime}\left(t \tilde{w}_{t}\right)+\beta_{6}^{\prime}\left(t^{2} \tilde{w}_{t}\right)+\beta_{7}^{\prime}\left(t^{3} \tilde{w}_{t}\right)\right\} G_{c},
$$

where $G_{\gamma_{1}}, G_{\gamma_{2}}$, and $G_{c}$ are the derivatives of $G\left(y_{t-d}, \tilde{\gamma}, h\left(\eta_{t}\right)\right)$ with respect to $\gamma_{1}, \gamma_{2}$ and $c$. With this notation, the estimators of $\frac{\partial \mathcal{L}_{t}}{\partial \gamma_{1}}, \frac{\partial \mathcal{L}_{t}}{\partial \gamma_{2}}$ and $\frac{\partial \mathcal{L}_{t}}{\partial c}$ are $\frac{\partial \hat{\mathcal{L}}_{t}}{\partial \gamma_{1}}=\frac{1}{\hat{\sigma}^{2}} u_{t} \hat{G}_{\gamma_{1}}, \frac{\partial \hat{\mathcal{L}}_{t}}{\partial \gamma_{2}}=\frac{1}{\hat{\sigma}^{2}} u_{t} \hat{G}_{\gamma_{2}}, \frac{\partial \hat{\mathcal{L}}_{t}}{\partial c}=\frac{1}{\hat{\sigma}^{2}} u_{t} \hat{G}_{c}$ respectively, so that: $\left.\hat{z}_{t}=\left(1, \bar{z}_{t}^{\prime}, \tilde{z}_{t}^{\prime} \hat{G}_{\left(y_{t-d}\right.} ; \cdot\right), \hat{G}_{\gamma_{1}}, \hat{G}_{\gamma_{2}}, \hat{G}_{\gamma_{c}}\right)^{\prime}$ and $\hat{\mathrm{u}}_{t}=\left(t \bar{z}_{t}^{\prime}, t^{2} \bar{z}_{t}^{\prime}, t^{3} \bar{z}_{t}^{\prime}, t \tilde{z}_{t}^{\prime} \hat{G}\left(y_{t-d}, \cdot\right), t^{2} \tilde{z}_{t}^{\prime} \hat{G}\left(y_{t-d}, \cdot\right), t^{3} \tilde{z}_{t}^{\prime} \hat{G}\left(y_{t-d}, \cdot\right)\right)$. Like in the symmetric case, under $\mathcal{H}_{0}$, the statistic Eq. (6A) is asymptotically distributed as a $\chi^{2}$ with $3(k+l)$ degrees of freedom, whereas the equivalent $F$-distribution has $3(k+l)$ and $T-4(k+l)-2$ degrees of freedom (the statistic is denoted $\left.L M_{3}\right)$. The following rule is used: if $\mathcal{H}_{1}$ is Eq. (11A) with transition function $G_{3}$, then Eq. (6A) is based on Eq. (15A) assuming $\beta_{3}=0$ and $\beta_{7}=0$ (statistic $L M_{2}$ ) and, if the same alternative hypothesis has the transition function $G_{2}$, the test is based on Eq. (15A), assuming $\beta_{2}=\beta_{3}=0$ and $\beta_{6}=\beta_{7}=0$ (statistic $L M_{2}$ ).

\section{References}

Abraham, J., Hendershott, P., 1996. Bubbles in metropolitan housing markets. J. Hous. Res. 7, 191-207.

Balcilar, M., Gupta, R., Miller, S.M., 2015. The out-of-sample forecasting performance of non-linear models of regional housing prices in the U.S. Appl. Econ. http://dx.doi.org/10.1080/00036846.2015.1005814.

Canepa, A., Zanetti Chini, E., 2015. Dynamic asymmetries in house price cycles: a generalized smooth transition model. Working Paper No. 15-23. Brunel University, London.

Case, K.E., Shiller, R.J., 1989. The efficiency of the market for single-family homes. Am. Econ. Rev. 79, $125-137$.

Case, K.E., Shiller, R.J., 2003. Is there a bubble in the housing market?. Brook. Pap. Econ. Act. 2, $299-362$.

Chan, K., Tong, H., 1986. On estimating thresholds in autoregressive models. J. Time Ser. Anal. 7, 178-190.

Crawford, G., Fratantoni, M., 2003. Assessing the forecasting performance of regime-switching, ARIMA and GARCH models of house prices. Real Estate Econ. 31, $223-243$.

Eitrheim, O., Teräsvirta, T., 1996. Testing the adequacy of smooth transition autoregressive models. J. Econ. 74, 59-75.

Holly, S., Pesaran, M.H., Yamagata, T., 2010. A spatio-temporal model of house prices in the USA. J. Econ. 158, 160-173.

Holly, S., Peseran, M.H., Yamagata, T., 2011. Spatial and temporal diffusion of house prices in the UK. J. Urban Econ. 69, 2-23.

Kim, S., Bhattacharya, R., 2009. Regional housing prices in the USA: an empirical investigation of nonlinearity. J. Real Estate Financ. Econ. 38, 443-460.

Leybourne, S., Newbold, P., Vougas, D., 1998. Unit roots and smooth transitions. 19, 83-97.

Lundbergh, S., Teräsvirta, T., 2006. A time series model for an exchange rate in a target zone with applications. J. Econ. 131, 579-609.

Luukkonen, R., Saikkonen, P., Teräsvirta, T., 1988. Testing linearity against smooth transition autoregressive models. Biometrika 75, $491-499$.

Muellbauer, J., Murphy, A., 1997. Booms and busts in the U.K. Housing market. Econ. J. 107, 1701-1727.

Sichel, D., 1993. Business cycle asymmetry: a deeper look. Econ. Inq. 31, 224-236.

Sollis, R., Leybourne, S., Newbold, P., 1999. Unit roots and asymmetric smooth transitions. J. Time Ser. Anal. 20, 671-677.

Sollis, R., Leybourne, S., Newbold, P., 2002. Tests for symmetric and asymmetric nonlinear mean reversion in real exchange rates. J. Money Credit Bank. 34, $686-700$.

Stukel, T., 1988. Generalized logistic models. J. Am. Stat. Assoc. 83 (402), 426-431.

Teräsvirta, T., 1994. Specification, estimation and evaluation of smooth transition autoregressive models. J. Am. Stat. Assoc. 89, $208-218$.

Tong, H., 1983. Threshold Models in Non-linear Time Series Analysis, No 21 in Lecture Notes in Statistics. Springer-Verlag, New York.

Tsay, R., 1989. Testing and modeling threshold autoregressive processes. J. Am. Stat. Assoc. 84, 231-240.

Zanetti Chini, E., 2014. Generalizing smooth transition autoregression. CEIS Tor Vergata Research Paper Series, Vol. 11,Issue 13. 